\title{
Physics of Laser in Contemporary Visual Arts: the research protocol
}

\author{
Diaa Ahmed Mohamed Ahmedien $\neq, \S$ \\ ‡ University of Bern, Bern, Switzerland \\ § University of Applied sciences, Bern, Switzerland
}

Corresponding author: Diaa Ahmed Mohamed Ahmedien (diaa.ahmedien@gmail.com)

Reviewable $\quad \mathrm{v} 1$

Received: 10 Nov 2016 | Published: 25 Nov 2016

Citation: Ahmedien D (2016) Physics of Laser in Contemporary Visual Arts: the research protocol. Research Ideas and Outcomes 2: e11150. https://doi.org/10.3897/rio.2.e11150

\begin{abstract}
This protocol articulates an ongoing PhD thesis in Laser Art as an independent artistic trend including its history, classification criteria, philosophical and aesthetical aspects. Via several analytical studies theoretically and practically, the thesis is going to prove that laser art actively contributes, as an independent artistic trend, to change the conceptual definition of the artistic material. On the other hand, it bridges the gap between the artistic context and the technical issues, in which the conceptual values became fully integrated into the physical properties of the medium (laser beam), therefore it became impossible to separate the idea and the material of the artwork from each other. Besides, the thesis reveals the potential hidden conceptual and philosophical dimensions of the holographic art as one of the most important branches of laser art. In advanced step, the research suggestes a new model of interactive holographic art based on neural controlling system, and how this advanced approach can lead us towards a new kind of the aesthetic values, in which participants effectively contribute neurologically to constituting the artwork.
\end{abstract}

\section{Keywords}

Laser Art; New-Media arts; sciences of visual arts, holographic art; neural holography; new aesthetics 


\section{Problem staement, objectives, importance}

\section{Research questions:}

According to the state of the previous research, it became obvious that there are knowledge gaps in Laser art because it is still outside the theorizing circle (Analysis, Classification, Documentation). This problem hamper progress in the artistic practical procedures as a result of the missing the conceptual approaches. Accordingly, this research tries to answer four questions:

1. How can the scientific procedures in Laser Art contribute to reformulate a new key to $21^{\text {st }}$ century aesthetics in contemporary visual arts?

2. To what extent physics of laser beam has a significant role to change the nature of light art through contemporary visual arts?

3. How far some of theoretical theories can reinterpret the laser artworks as an independent visual artistic trend?

4. Can some kind of advanced interactive technologies represent new approaches towards re-defining laser beam as an artistic element and material in the light art?

\section{Hypothesis:}

1. The physics of the Laser beam has significantly contributed to change the nature of light into a Hyper-Material in the contemporary visual arts technically and conceptually.

2. The scientific processes and technological techniques have significant effects on the nature of the aesthetical values in laser artworks.

3. The theory of information and the theory of communication can reinterpret laser artworks quantitatively and qualitatively" as an independent contemporary artistic trend.

4. Holographic Neural Game can represent a revolutionary assumption in laser art by using neural inputs to build an interactive artwork through a holographic environment.

\section{Objectives:}

1. Investigate and compare the history of visible light usage in artworks before and after the availability of laser light through the historical relationship among art, science, and technology technically and conceptually.

2. Evaluate the role of contemporary theories to explain, classify, and document laser artworks conceptually and technically.

3. Analyze the new artistic and aesthetical values, which are emerged as a result, from the use of laser light in artworks as an extraordinary element and material.

4. Examine some of interactive approaches technically to design an interactive virtual environment as an advanced level of interactive holography. 


\section{Importance:}

1. Confirming solid principles of laser arts as andependent and advanced level in contemporary visual art trends.

2. Enrich laser art technically and conceptually as a one of the important branches in sciences of visual arts.

3. Support new advanced scientific methodologies in sciences of visual arts by laser artworks that can melt artistic, scientific, and technological concepts in one artwork.

4. Improve some of technical approaches in sciences of visual arts as a significant guide to analyze laser artworks.

5. Emphasize the deep philosophical and technical knowledge of sciences of visual arts generally.

6. Reinterpret the relationship among art, science, and technology by new understanding of contemporary visual arts.

\section{Project Overview}

Since the middle of the $20^{\text {th }}$ century, the scientific and artistic communities have both attempted to understand the beginning of the universe, the origin of the human being, consciousness, and human brain in order to decode our potential future, our identities, our cultures and civilizations based on the latest scientific mutations and discoveries. Accordingly, the 'visual arts', as an important branch of humanities, is no longer an isolated domain, but it developed a strong relationship with other scientific disciplines (Wilson 2003) This could be considered as one of the most important characteristics in some contemporary visual arts trends, which are reflected in a renewed and strong sense of affinity between art, science and technology (Canaria 2009) After all, the relationship among art, science, and technology (AST) has extended roots through the history of art (Hughes 2006). The difference in our contemporary era is represented in several paradigm shifts that have broken the dividing line among scientific and artistic specializations by conducting cutting-edge research cross a wide range of disciplines. Consequently, in some cases it became difficult to redefine sharp boundaries among some of the artistic and scientific fields (Shanken 2002). So, 'interdisciplinarity' as a term reflects a new method that has already dominated scientific and artistic activities. Due to those paradigm shifts that happened in the scientific methodological practices through many artistic and scientific domains, in his book entitled "Art and science now", Stephen Wilson describes accurately the current phase of the unity among art, science, and technology and its effects on the nature of the artistic creation processes in some contemporary experimental artistic trends:

"In the $21^{\text {st }}$ century, some of the most dynamic works of art are being produced not in the studio but in the laboratory, where artists probe cultural, philosophical and social questions connected with cutting-edge scientific and technological research. Their work ranges across disciplines - microbiology, the physical sciences, information technologies, human biology and living system, kinetics and robotics- taking in 
everything from eugenics and climate change to virtual reality and artificial intelligence" (Wright 2010).

In fact, Wilson's description of the contemporary visual arts trends is confirmed by what Roy Ascott said about the contemporary visual arts when he emphasized the relationship between art science and technology in visual arts has been divided into three phases: The first and the second have shared properties; most important of them are reliance on the use of technology either directly or indirectly in the artworks, and several sciences applications had been expanded to be used in many visual arts trends (Popper 1993).

Nevertheless, the contemporary documented roots of the third phase of the relationship between art, sciences and technology may be detected in the C. P. Snow's famous annual Rede lectures at Cambridge on May $7^{\text {th }}, 1959$. These lectures were combined in a book entitled "The Two Cultures and the Scientific Revolution" (Shanken 2002). In the second edition of This book, in 1963, Snow added a new essay entitled: "The Two Cultures: $A$ Second Look". In that essay he suggested a 'Third Culture' as a new term that would emerge and close the gap between artistes and scientists, where the sciences were not used in an unlimited way, rather, the art and sciences became two sides of one coin. Consequently, The artworks are achieved in laboratories, and artists and scientists moved along a common path, to the extent that some of the artistic trends were classified by some kinds of scientific techniques that are used to achieve them, for instance: (Laser Art physics applications in visual arts), (Bio Art - Biological sciences in visual arts) and (Mechatronic Art - Engineering applications in visual arts). (Molnar 1997). All of those three visual artistic trends belong to the 'Processes art' historically, in which the conceptual core of the artwork is constituted in the processes performed beyond the visual form or even the physical form of the artwork itself (NAGAI 2000).

'laser light' as an artwork may have the biggest chance for expansion, more than the other kinds of these trends, because it represents a unique Hyper-Material due to its 'spatial' and 'temporal' 'coherence' leading to 'speckle' and allowing signal processing, 'interference patterns', very 'narrow beam width' and very 'narrow line width' which leads to strong and pure colors (the scientific properties became artistic advantages; (Oliveira and Richardson 2013).

On the other hand, the use of laser demands high skills and expensive equipment which forces the artist to perform their artworks in cooperation with scientists inside their labs. Besides, artistically it has extraordinary ability to interact with different environments and audiences (Szanto 2015).

In terms of theoretical documentation in 'laser art', we can see most of references explain and analyze laser artworks in the same way that is usually used to analyze other visual arts trends and the same criteria without taking into account the special case of laser artworks. Few studies highlighted laser art in a more suitable position, the common characteristic in all of these studies that they were written under the same title (art and science), and the laser artworks were a part of them (Bjelkhagen 2013). 
Conceptually, the current research focuses on the laser light itself as an artwork, the research wants to elucidate the historical roots of laser artworks conceptually and technically. the current work will focus on the full period from the invention of the laser in 1960 up to the present (Soskind et al. 2015).

However, applications in which lasers were a source of illumination, like laser shows, are not a part of the current study. The research attempts to achieve new approaches to explain and document new artistic and aesthetical values, which are a result of laser light usage in contemporary artworks conceptually and practically. The theoretical outcome of the research should lead to a new classification for types of laser artworks, therefore enriching the contemporary visual arts trends by accurate analytical studies and practical experiments in laser arts (Ahmedien 2016).

For better understanding of laser artworks, several experiments were done in the current research comprising of three parts; the first part was conducted in a holographic lab to produce an analogue holographic recorded image and analyze the result to document the scientific processes and their effect on the aesthetical values by comparing the holographic image to a normal image. The second part was lead to experimental studies in light and interactive techniques and how advanced technology can redefine the concept of light through the contemporary visual arts trends. The third part which is only a concept of a potential project: the design of an interactive system by using neural controlling techniques to create an advanced virtual environment as a neural holographic game that can interact with audience automatically or can be controlled by the audience neurologically.

\section{Implementation}

\section{Methodology}

\section{Theoretical framework}

The Theoretical Framework is consisted of two axes; the historical axis and conceptual axis as follows:

\section{Historical Axis:}

- $\quad$ analytical studies of the previous literatures

- Drawing analytical diagrams to summarize progress processes in light art before laser through visual arts and related domains.

- Analytical studies of the history of the theory of laser action.

Conceptual Axis:

- Analytical studies of the formation theories by laser beam in visual arts.

- Analytical studies to document the new artistic and aesthetical values, which have existed as a result of laser light usage in contemporary artworks. 
- Analytical studies of some of leading contemporary theories to classify laser artworks.

\section{Practical Framework}

The Practical Frame is consisted of two axes; the experimental studies and the final project as follows:

\section{Experimental Studies:}

- Experimental studies to classify laser artworks technically and conceptually.

- Experimental studies in object building techniques (Hologram).

- analytical studies in interactive techniques.

Final Practical Project:

- designing experimental setup to recorded neural responses holographically.

- Designing an interactive system in neural based

- Designing a setup of Neural Holographic Game.

\section{Structural Mode}

the accumulation system was used to constitute the manuscript. Through seven separate Research that have been partly published internationally, this thesis summarizes the main ideas of theses research including the final findings.

\section{Structural Pattern}

This thesis follows the compromise pattern in its structure: structural Matrix $\underline{\mathbf{B}}$ in the $\mathrm{PhD}$ guide to style, 2nd end, Houndmills, Basingstoke, Hampshire: Palgrave Macmillan (Hammersley 2003). The thesis has been written using Harvard style (Harvard - Cite Them Right 9th Edition). Accordingly, the research will be divided into 5 chapters as the thesis' flow chart shows Fig. 1.

\section{Data balance:}

Depending on the compromise pattern, the research keeps palance by dividing data into inputs, outputs and the core of the research processing as Fig. 2 shows.

\section{Work plan}

The research will follow a custom-designed, logical framework matrix (LFM model) to determine to what extent the current research questions can be answered, besides, to illustrate a set of physical indicators that can refer to a potential positive result as Figs 3,4 illustrate. 


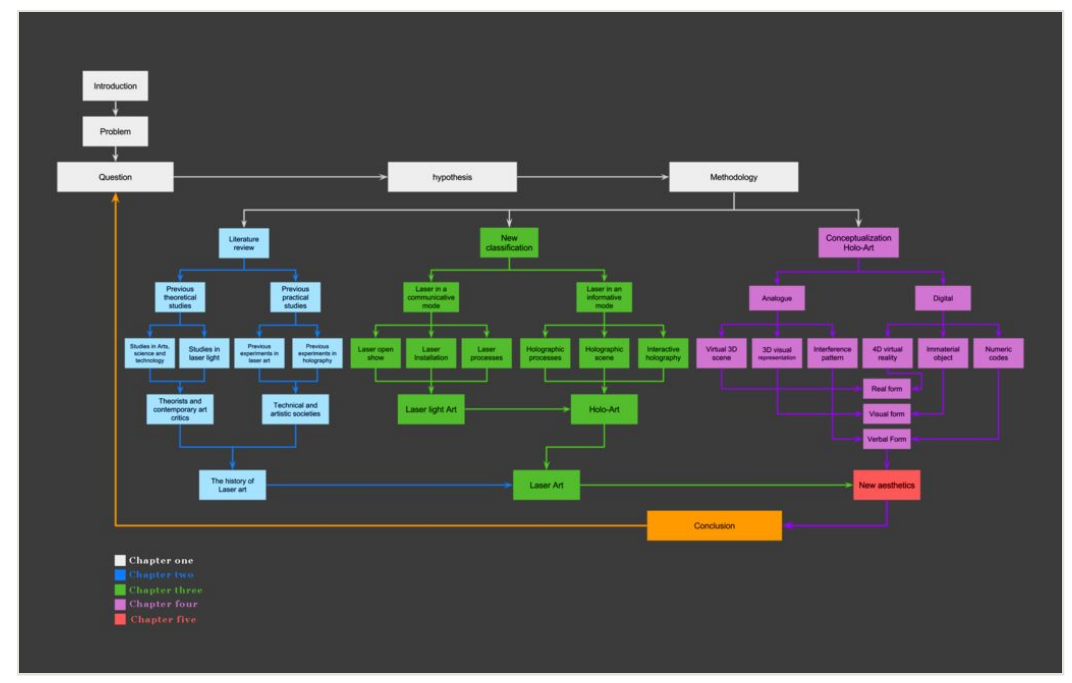

Figure 1.

the flow chart of the thisis that shows thesis' chapters depending on the research methodology

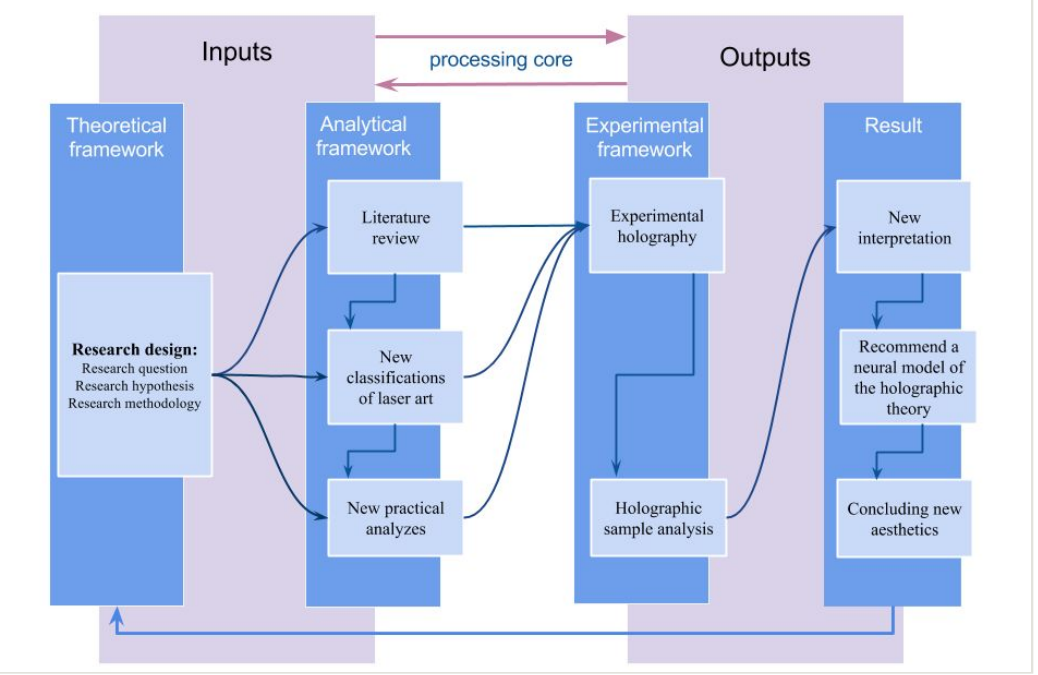

Figure 2.

the graph illustrates the Data flow through the methodology designed in order to control the circle of the data processing from the inputs into the outputs and the processes in the middle through all research processes. 


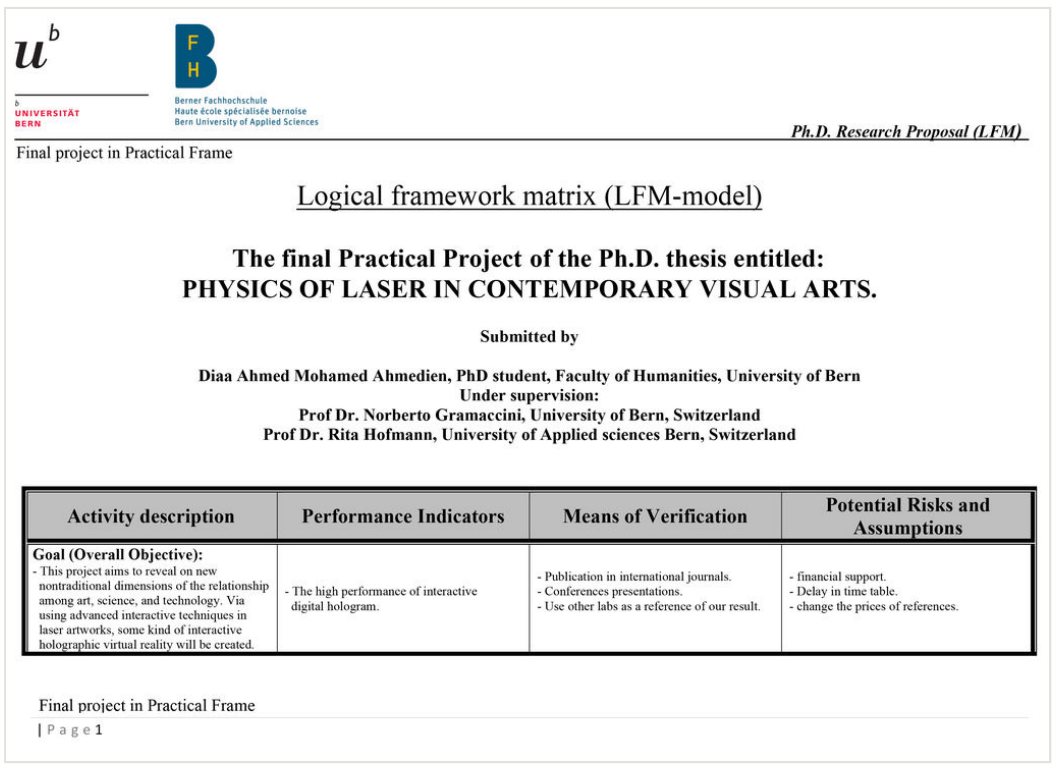

Figure 3.

Logical Framework Matrix (LFM model) part 1

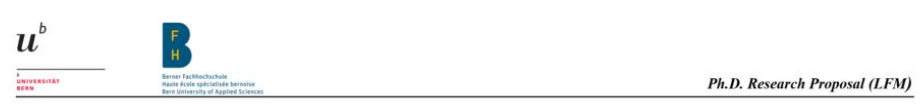

\begin{tabular}{|c|c|c|c|}
\hline Activity description & Performance Indicators & Means of Verification & $\begin{array}{l}\text { Potential Risks and } \\
\text { Assumptions }\end{array}$ \\
\hline 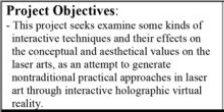 & 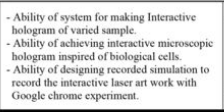 & 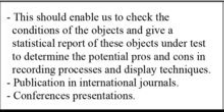 & $\begin{array}{l}\text { - Avaiability of necessury components and } \\
\text { requirements of system. } \\
\text { - Periedic maintenane } \& \text { calibration of the } \\
\text { laser systems- Lack of taime } \\
\text { - Training prolongation }\end{array}$ \\
\hline 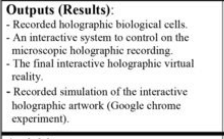 & 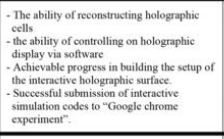 & 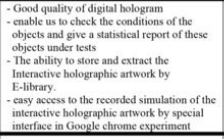 & 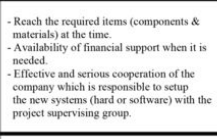 \\
\hline 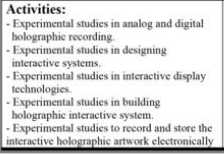 & $\begin{array}{l}\text { - Collect the practical references } \\
\text { - Collect the equipment } \\
\text { - System integration } \\
\text { - Achievement the high quality }\end{array}$ & $\begin{array}{l}\text { - Regular monitoring of ongoing activitics an } \\
\text { compatibihity with the project time planc. } \\
\text { - Regular reporting }\end{array}$ & $\begin{array}{l}\text { 1- Delay in equipment \& materials delivery. } \\
\text { 2- Prices will not change in the nexi } 6 \text { months } \\
\text { 3. Decla in inancial support: } \\
\text { 4- Continues capacity building }\end{array}$ \\
\hline \multicolumn{2}{|c|}{ Prof Dr. Norberto Gramaccini } & \multicolumn{2}{|c|}{ Prof Dr. Rita Hofmann } \\
\hline
\end{tabular}

Figure 4.

Logical Framework Matrix (LFM model) part 2 


\section{Details for replicability and reproducibility}

The research in its practical frame can be re-examined in holographic labs under the instructions of the intellectual property laws at the university of Bern.

\section{Timeline}

The thesis' time table Fig. 5 illustrates the action plan that can be followed to achieve the research within about three years.

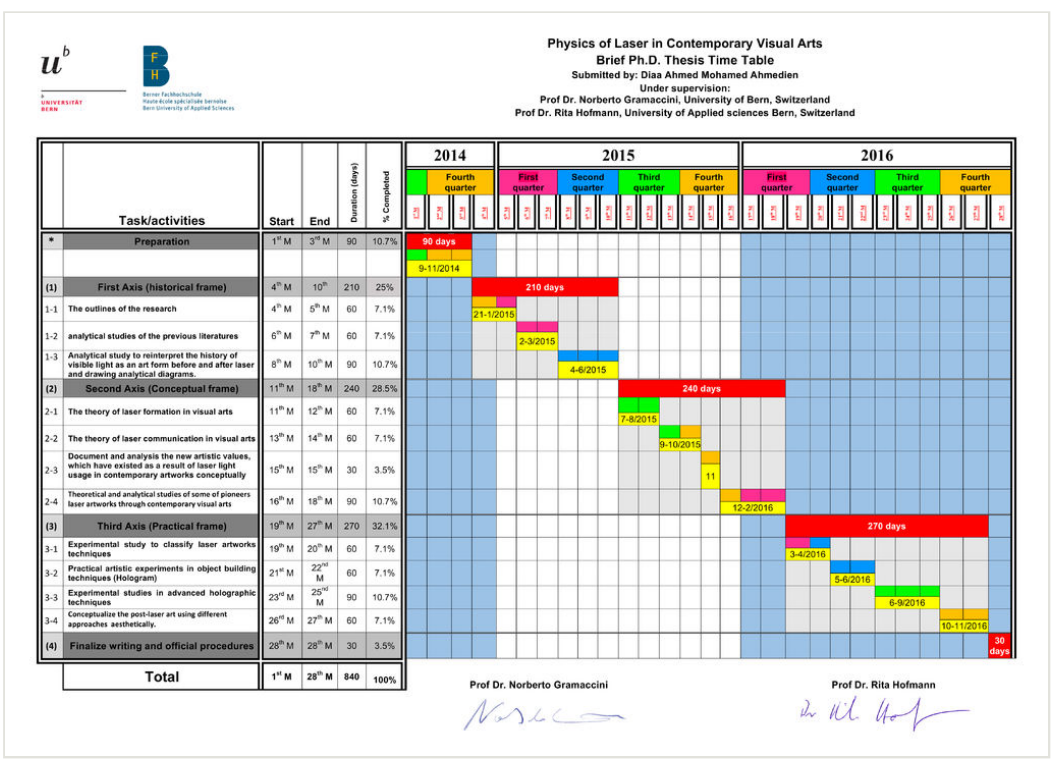

Figure 5.

Thesis' time table within 3 years

\section{Funding program}

Study full PhD programe in sciences of visual arts and new-media arts.

\section{Grant title}

Egyptian Government Excellence Scholarship. 


\section{Hosting institution}

- Faculty of Humanities, Visual arts Dept., Bern University, Switzerland

- School of Arts, Universtiy of Applied sciences, Bern, Switzerland

\section{Ethics and security}

The entire project theoretically and practically undergoes to all Ethics and security laws of scientific research in the University of Bern.

\section{Author contributions}

Diaa Ahmed Mohamed Ahmedien

\section{Conflicts of interest}

The authour has no any confliect of interest

\section{References}

- $\quad$ Ahmedien DAM (2016) Reactivating the Neural Dimension Role in Interactive Arts. Leonardo [In Englis]. https://doi.org/10.1162/leon a 01376

- Bjelkhagen HI (2013) Ultra-realistic 3-D imaging based on colour holography. Journal of Physics: Conference Series 415: 012023. https:// doi.org/10.1088/1742-6596/415/1/012023

- Canaria C (2009) Data + Art: science and art in the age of information. Disease Models \& Mechanisms 2: 538-540. https://doi.org/10.1242/dmm.004291

- Hammersley P (2003) Authoring a PhD Patrick Dunleavy Authoring a PhD Palgrave Macmillan No of pages: $300 £ 16.991403905843$ 1403905843. Nurse Researcher 11

(1): 77-78. https://doi.org/10.7748/nr.11.1.77.s7

- Hughes G (2006) Power's Script: or, Jenny Holzer's Art after 'Art after Philosophy'. Oxford Art Journal 29 (3): 419-440. https://doi.org/10.1093/oxarti/kcl017

- Molnar F (1997) A Science of Vision for Visual Art. Leonardo 30 (3): 225. https:// doi.org/10.2307/1576454

- $\quad$ NAGAI H (2000) Recent Laser Technology and Its Applications to Material Processing. The Review of Laser Engineering 28 (1): 2-2. https://doi.org/10.2184/lsj.28.2

- Oliveira S, Richardson M (2013) The future of holographic technologies and their use by artists. Journal of Physics: Conference Series 415: 012007. https://

doi.org/10.1088/1742-6596/415/1/012007 
- $\quad$ Popper F (1993) The Place of High-Technology Art in the Contemporary Art Scene. Leonardo 26 (1): 65. https://doi.org/10.2307/1575783

- Shanken E (2002) Art in the Information Age: Technology and Conceptual Art. Leonardo 35 (4): 433-438. https://doi.org/10.1162/002409402760181259

- Soskind M, Soskind R, Soskind $Y(2015)$ Shaping propagation invariant laser beams. Optical Engineering 54 (11): 111309. https://doi.org/10.1117/1.oe.54.11.111309

- Szanto A (2015) Reporting the Arts II [2003]. ICPSR - Interuniversity Consortium for Political and Social Research 00 https://doi.org/10.3886/ICPSR35590.V1

- Wilson S (2003) Commentary on "art in the Information Age: Technology and Conceptual Art”. Leonardo 36 (3): 237-237. https:// doi.org/10.1162/002409403321921488

- Wright E (2010) Art+Science Now - How Scientific Research and Technological Innovation Are Becoming Key to 21st- Century Aesthetics by Stephen Wilson. The Art Book 17 (4): 63-64. https://doi.org/10.1111/j.1467-8357.2010.01137_15.x 